\title{
Guillain-Barre syndrome after lumbar epidural block
}

\author{
Myo Seop Yun, Yong-Hyun Cho, Dong-Hyun Lee, and Hyung-Woo Lim \\ Department of Anesthesiology and Pain Medicine, Seoul Sungsim General Hospital, Seoul, Korea
}

Guillain-Barre syndrome (GBS) is an acute demyelinating polyneuropathy characterized by weakening of muscular strength on both sides of the body and ascending paralysis of the body, and displays a prevalence rate of $0.6-4$ out of 100,000 persons per year [1]. Clinical appearance mostly begins with weakening of muscular strength in the distally located lower limb and progresses to the proximal muscles including the trunk, neck, and face over several days [2]. Here, we report a case of GBS that occurred after a lumbar epidural block along with a literature review.

A 26-year-old man who visited the pain clinic at our hospital presented with an aggravation of radiating pain to the right lower limb. The patient was diagnosed with lumbar herniated intervertebral disc between the 5th lumbar and 1st sacral vertebrate with magnetic resonance imaging (MRI) done at our hospital about a year ago. The patient was injected with a mixture consisting of $2 \mathrm{ml}$ of $1 \%$ mepivacaine, $1 \mathrm{ml}$ of triamcinolone $(40 \mathrm{mg} / \mathrm{ml})$, and $5 \mathrm{ml}$ of normal saline into the epidural space with a 22-gauge epidural Tuohy needle between the 5th lumbar and 1st sacral vertebrate. Although the radiating pain to the right lower limb improved, he revisited the emergency ward of our hospital with a feeling of powerlessness in both lower limbs that increasingly worsened 4 days after the epidural block. During the motor test, grade III-IV hip joint flexion, grade IV hip joint extension on both sides, grade II knee joint flexion, grade IV knee joint extension on both sides, and grade II dorsi-flexion of the ankle joint on both sides were observed. Reflex test demonstrated findings of areflexia in the knees and Achilles tendon on both sides. The patient had diarrhea several times 10 days before visiting the pain clinic at our hospital, which improved without any particular treatment. An MRI was taken to reconfirm the extent of the lumbar herniated intervertebral disc, which displayed findings of reduction in the extent of the lumbar herniated intervertebral disc compared to the MRI taken a year ago. While the test was being done, the patient complained of a sensory abnormality around his lips, and the patient was transferred to the department of neurology at a higher-ranking hospital with suspicion of GBS. Cerebrospinal fluid (CSF) analysis resulted in findings of increased levels of proteins to $121 \mathrm{mg} / \mathrm{dl}$ (normal level, $15-45 \mathrm{mg} / \mathrm{dl}$ ). In the nerve conduction test, terminal latency in the peroneal nerve and posterior tibial nerve on both sides was prolonged while compound muscle action potential amplitude and conduction velocity were within normal range. F-wave latency was prolonged in the posterior tibial nerve. Having been diagnosed with GBS, the patient was administered immunoglobulin for 5 days. The patient was discharged after having shown improvement after 10 days of treatment in the hospital.

If it is GBS, then an immune reaction is the cause of this disease. Approximately $2 / 3$ of patients affected by this disease have a past history of infection in the respiratory or gastrointestinal system 6 weeks prior to the occurrence of polyneuropathy [1]. For a diagnosis of GBS, findings of a gradual lowering of muscular strength in the upper and lower limbs within 4 weeks accompanied by areflexia under physical examination are essential. CSF analysis could be useful. In addition, findings such as a reduction in the motor nerve conduction velocity and extension of the F-wave latency could be found with a nerve conduction test. Intravenous immunoglobulin and plasma exchange are efficacious treatments. Supportive care during and following hospitalization is also crucial [2]. Steiner et al. [3]

Corresponding author: Yong-Hyun Cho, M.D., Department of Anesthesiology and Pain Medicine, Seoul Sungsim General Hospital, 40-12, Cheongnyangni-dong, Dongdaemun-gu, Seoul 131-868, Korea. Tel: 82-2-966-1616, Fax: 82-2-968-2394, E-mail: anesthecho@naver.com (c) This is an open-access article distributed under the terms of the Creative Commons Attribution Non-Commercial License (http:// creativecommons.org/licenses/by-nc/3.0/), which permits unrestricted non-commercial use, distribution, and reproduction in any medium, provided the original work is properly cited. 
argued that the interaction between anesthetics and peripheral nerve myelin or local trauma to the roots could trigger a cascade of immunological events that result in demyelinating neuropathy. The researchers reported that the drugs used for regional anesthesia contain an aromatic ring that renders them lipid-soluble allowing them to pass through the myelin of the peripheral nerves and axons, thereby inducing damages to the nerves through the interaction between the anesthetics and the myelin of the peripheral nerves. Segerstrom and Miller [4] reported that stress could change the cellular or humoral immunity. An increase in the concentration of cytotoxic T cells and natural killer cells in the peripheral circulation was observed within several minutes of sudden mental stress, and they explained the damages to the peripheral nerves could be caused by inducing an influx of lymphocytes from the lymphoid organs into the blood vessels due to such changes.

In our case, clinical symptoms of GBS appeared after epidural anesthesia. Accordingly, the possibility of damage to the peripheral nerves through the interaction between the anesthetics and the myelin of the peripheral nerves, and direct injuries to the nerve roots can be considered as one possibilty. In addition, we could consider the possibility that mental and physical stress due to the epidural block, which is an invasive procedure, could have imparted an effect on the immune system.

Based on the past history of the patient having diarrhea several times 10 days prior to his visit to our hospital, it seems he had an infection of the gastrointestinal system, and the possibility of GBS being induced due to this infection cannot be ruled out. In addition, we can consider the possibility that infection of the gastrointestinal system and changes in the immune system by the mental and physical stress imparted at the time of the epidural block complexly operated to accelerate the progression of GBS.

Although we cannot conclude that the epidural block is the cause of GBS in this case, one must take accurate medical histories of and execute physical examinations on patients who display reduced muscular strength on both sides of the body and ascending paralysis of the body after epidural block, since GBS can progress quickly and be fatal. Moreover, the distinction of GBS from other diseases and early treatment of GBS through exhaustive tests including CSF analysis, nerve conduction test, and MRI would be helpful in lowering the mortality and improving the quality of life.

\section{References}

1. Hughes RA, Cornblath DR. Guillain-Barre syndrome. Lancet 2005; 366: 1653-66.

2. Burns TM. Guillain-Barre syndrome. Semin Neurol 2008; 28: 15267.

3. Steiner I, Argov Z, Cahan C, Abramsky O. Guillain-Barre syndrome after epidural anesthesia: direct nerve root damage may trigger disease. Neurology 1985; 35: 1473-5.

4. Segerstrom SC, Miller GE. Psychological stress and the human immune system: a meta-analytic study of 30 years of inquiry. Psychol Bull 2004; 130: 601-30. 